\title{
Novel AMBRA1-ALK fusion identified by next-generation sequencing in advanced gallbladder cancer responds to crizotinib: a case report
}

\author{
Yuling Zhou ${ }^{1,2}$, Analyn Lizaso ${ }^{3}$, Xinru Mao ${ }^{3}$, Nong Yang ${ }^{1}$, Yongchang Zhang ${ }^{1}$ \\ ${ }^{1}$ Department of Medical Oncology, Lung Cancer and Gastrointestinal Unit, Hunan Cancer Hospital/The Affiliated Cancer Hospital of Xiangya \\ School of Medicine, Central South University, Changsha, China; ${ }^{2}$ Graduate Schools, University of South China, Hengyang, China; ${ }^{3}$ Burning Rock \\ Biotech, Guangzhou, China \\ Correspondence to: Dr. Yongchang Zhang. Department of Medical Oncology, Lung Cancer and Gastrointestinal Unit, Hunan Cancer Hospital/The \\ Affiliated Cancer Hospital of Xiangya School of Medicine, Central South University, Changsha, China. Email: zhangyongchang@csu.edu.cn.
}

\begin{abstract}
Gallbladder cancer (GBC) is the most aggressive malignancy of the biliary tract with poor prognosis. Several targetable genetic alterations have been identified in GBC; however, responses to targeted therapy are disappointing. We report a case of a 58-year-old Chinese woman with GBC who was detected with a novel $A L K$ genomic rearrangement and received crizotinib after progression from first-line chemotherapy. The patient was diagnosed with stage IV adenocarcinoma of the neck of the gallbladder and received oxaliplatin combined with capecitabine as first-line therapy. After four cycles of this chemotherapy regimen, the patient started to show obstructive jaundice, and progressive disease was evaluated. Biliary drainage surgery was performed to alleviate the symptoms of obstructive jaundice. Upon referral to our department, her archived tissue samples were submitted for next-generation sequencing (Burning Rock Biotech) and immunohistochemistry, which identified the presence of a novel AMBRA1-ALK rearrangement and ALK overexpression, respectively. Oral crizotinib was administered achieving partial response within two cycles of treatment, which lasted for 7 months. AMBRA1-ALK has not been previously reported in any solid tumors and its sensitivity to crizotinib is not well characterized. Moreover, ALK alterations have been rarely reported for GBC. This case suggests that a subset of GBC might be driven by aberrant ALK signaling, which could potentially be explored as a biomarker of therapeutic response to ALK inhibitors in GBC. Moreover, our case report contributes an incremental step in understanding the genetic heterogeneity in GBC and provides clinical evidence of the utility of next-generation sequencing in exploring actionable mutations to expand treatment choices in rare solid tumors including GBC.
\end{abstract}

Keywords: Targeted therapy; ALK rearrangement; biomarker; gallbladder cancer (GBC); crizotinib; case report

Submitted Jan 23, 2020. Accepted for publication Aug 07, 2020.

doi: 10.21037/atm-20-1007

View this article at: http://dx.doi.org/10.21037/atm-20-1007

\section{Introduction}

Gallbladder cancer (GBC) is the most common and most aggressive malignancy of the biliary tract (1). Owing to the insidious nature of GBC, it is usually diagnosed at an advanced stage leading to a dismal prognosis with a 5 -year survival rate of less than $5 \%$ for patients managed with cytotoxic chemotherapies $(2,3)$. With the development of nextgeneration sequencing (NGS), some key oncogenic pathways were found to be altered in GBC, including the epidermal growth factor receptor (EGFR) and vascular endothelial growth factor receptor (VEGFR) pathways (4-9). Several targeted agents are being explored in clinical trials for biliary cancers; however, results have so far been disappointing for patients with GBC (7,10-14). Effective targeted therapies and novel biomarkers for GBC are urgently needed to improve the prognosis of patients with GBC. 
A study has implicated protein overexpression of autophagy/Beclin-1 regulator 1 (AMBRA1), a crucial regulator of autophagy, in promoting cell invasion and poor prognosis in cholangiocarcinoma (15). Meanwhile, anaplastic lymphoma kinase (ALK), a tyrosine kinase receptor that regulates signaling pathways involved in normal cellular development, is one of the commonly deregulated oncogenes in solid tumors including non-smallcell lung cancer (NSCLC), and neuroblastomas (16-19). Crizotinib is an inhibitor that specifically binds to the ALK tyrosine kinase domain, which leads to the suppression of downstream signaling pathways (20). Crizotinib has been approved as the standard first-line therapy for $A L K$ positive advanced NSCLC. Although ALK overexpression has been reported for biliary tract cancers, $A L K$ fusions, particularly $A M B R A 1-A L K$ remain unreported. Moreover, reports on the efficacy of crizotinib in GBC harboring $A L K$ rearrangements or overexpression remains limited. Here, with the use of NGS, we identified a novel AMBRA1$A L K$ fusion in a patient with GBC who had remarkable therapeutic response to crizotinib.

We present the following article in accordance with the CARE reporting checklist (available at http://dx.doi. org/10.21037/atm-20-1007).

\section{Case presentation}

In January 2018, a 58-year-old female patient was admitted at Yueyang People's Hospital due to a space-occupying lesion located in the first hepatic hilar parenchyma observed through abdominal B-mode ultrasonography during routine physical examination. Abdominal computed tomography (CT), magnetic resonance imaging (MRI), and CT angiography demonstrated multiple soft lesions located in the neck of the gallbladder. The patient underwent laparotomy, which revealed a $5 \mathrm{~cm} \times 6 \mathrm{~cm}$ lump near the first hepatic hilum with invasion of the peritoneum and abdominal cavity; thus, only palliative biopsy was performed to obtain tissue specimens for differential diagnosis. Histological staining and immunochemistry (IHC) results were positive for CK7 (+), CK19 (++), Villin (+), MUC2 (+), and Ki67 (10\%), while negative for CK20, MUC5AC, and ALK. The diagnosis was concluded to be metastatic gallbladder adenocarcinoma. From February 2018, the patient was administered with oxaliplatin $\left(130 \mathrm{mg} / \mathrm{m}^{2}\right.$, ivgtt, once every 21 days) combined with capecitabine $\left(1,000 \mathrm{mg} / \mathrm{m}^{2}\right.$ orally twice daily for two weeks, every 21 days) as first-line therapy. After four cycles of this chemotherapy regimen, progressive disease was observed in May 2018, with increased tumor size in the neck of the gallbladder accompanied by obstructive jaundice. To address the obstruction, biliary drainage was performed and two WallFlex metal stents were implanted at Shanghai Oriental Hepatobiliary Hospital in May 2018.

She was then referred to Hunan Cancer Hospital for further management. A summary of her treatment history is illustrated in Figure 1. No family history of lung cancer, GBC, or other cancers was reported by the patient. MRI imaging at presentation indicated lesions on the gall bladder, presence of liver metastasis, and extensive omental metastasis as well as involvement of hilar lymph nodes, which made local therapy such as surgery and radiotherapy unsuitable for the management of her disease (Figure 1B). To explore potentially actionable mutations, NGS analysis was performed on her archival tissue biopsy specimen using a panel consisting of 520 genes (OncoSreen Plus, Burning Rock Biotech, Guangzhou, China). NGS identified a novel $A L K$ fusion gene involving the exon 13 of $A M B R A 1$ and exon 2 of $A L K$, which retains the tyrosine kinase domain of ALK (Figure $2 A$ ). No other genetic alteration was detected from the genes included in the gene panel. Ventana IHC also confirmed ALK protein overexpression (Figure 2B,C). The patient was then given crizotinib at a dose of $250 \mathrm{mg}$ orally twice daily starting from June 2018. MRI evaluation after 6 weeks of crizotinib therapy indicated partial response as evaluated by the investigators according to Response Evaluation Criteria in Solid Tumors version 1.1 (Figure 1C). No adverse events were experienced by the patient except for grade I appetite loss. The response was sustained for 7 months until disease progression was confirmed by the enlargement of the primary lesion. PDL1 (22-C3) immunohistochemistry testing of archived tissue biopsy specimen revealed low expression (1\%). She then received 2 cycles of pembrolizumab monotherapy at a dose of $200 \mathrm{mg}$ every 3 weeks without benefit. She was managed with best supportive care for 2 months until she passed away in May 2019 with overall survival of 16 months. All procedures performed in studies involving human participant were in accordance with the ethical standards of the institutional and national research committees, and with the Helsinki Declaration (as revised in 2013). Written informed consent was obtained from the patient for publication of this study and any accompanying images.

\section{Discussion}

With limited to no options for targeted therapeutics, 
A January 2018

May 2019

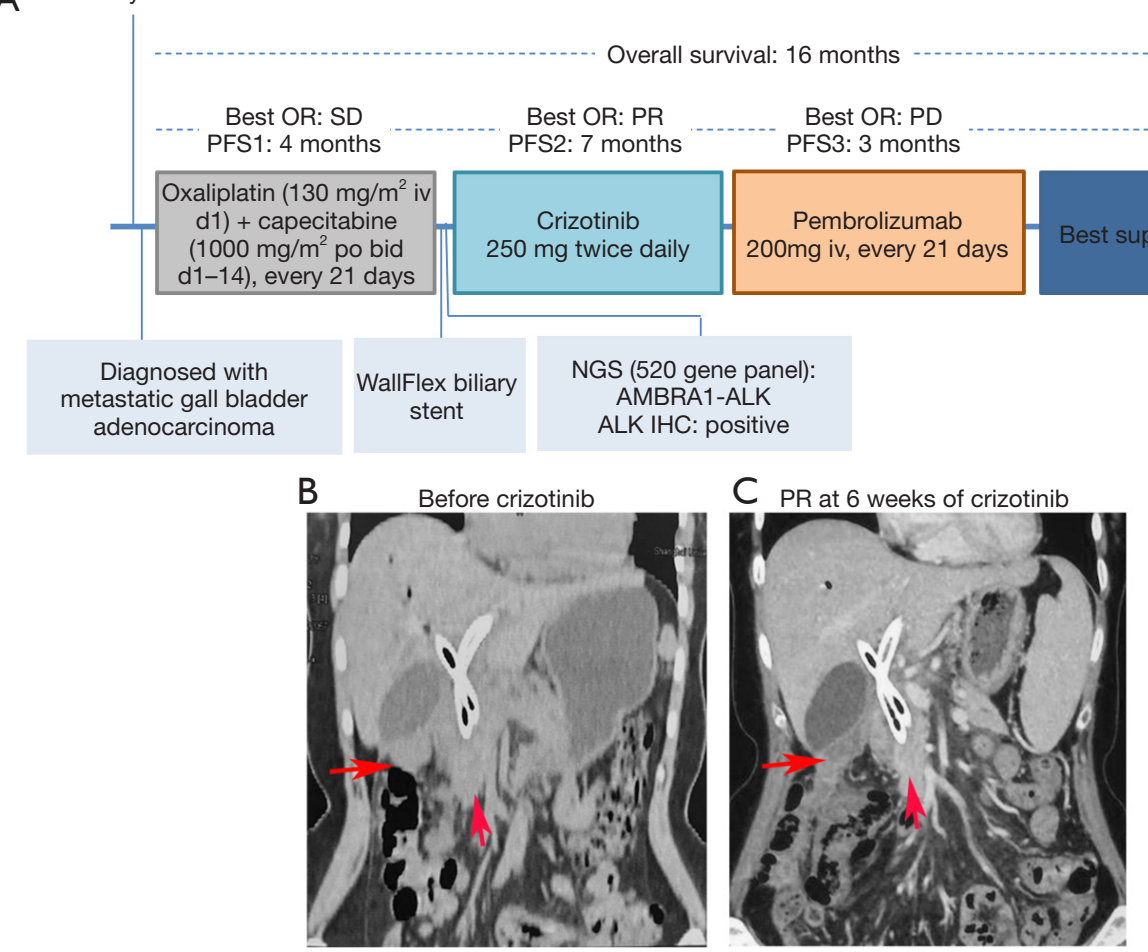

Figure 1 Treatment time-line and response. (A) A summary of the treatment history of the patient with advanced GBC. (B,C) Tumor evaluation by magnetic resonance imaging (MRI) before crizotinib (B) and after 6 weeks of crizotinib therapy demonstrating the significant shrinkage of the primary lesion evaluated as partial response (C). GBC, gallbladder cancer.

cytotoxic chemotherapy remains as the standard of care for patients with advanced GBC with limited duration of response between 3.1 and 5.6 months (21). To elucidate the molecular mechanisms of tumor development and identify potentially targetable genes, several studies have elucidated the genomic landscape of GBC and other types of biliary cancers and indicated the complexity and vast genetic heterogeneity of these tumors $(4-6,8,9)$. Consistently, numerous efforts have been invested in exploring the use of targeted drugs for advanced GBC. Unfortunately, clinical trials investigating the use of EGFR targeting therapies such as erlotinib, cetuximab, and panitumumab in combination with cytotoxic drugs have failed to show any benefit in terms of progression-free survival or overall survival as compared with chemotherapy alone $(10,12)$. Similarly, clinical trials of agents targeting VEGF and human epidermal growth factor receptor-2 (HER2) have been unsuccessful $(11,13)$. Hence, effective biomarkers to predict treatment response to targeted therapy are needed to be identified for GBC to improve their prognosis.

The $A M B R A 1$ gene encodes a protein that is involved in the crossroads between autophagy and apoptosis, and regulates the reciprocal conversion between autophagy and apoptosis to determine cell death or survival $(22,23)$; it is also involved in regulating the differentiation of regulatory T-cells and cell proliferation (23-25). This critical role of $A M B R A 1$ as a tumor suppressor gene implicates its genetic alterations in mediating tumorigenesis $(23,24)$. Consistently, AMBRA1 overexpression has been associated with poor prognosis in cholangiocarcinoma (15) Meanwhile, ALK overexpression has been reported in biliary tract cancers (26); however, the role of ALK in driving tumor development in GBC is not well understood. Moreover, AMBRA1-ALK has not been reported in any solid tumors and its sensitivity to ALK inhibitors is unexplored. In our case report, a novel $A M B R A 1-A L K$ was detected using NGS from a patient with advanced GBC after progression from first-line chemotherapy. The novel $A M B R A 1-A L K$ gene rearrangement identified from our patient encompassed exons 1 to 13 of $A M B R A 1$ fused to exon 2 to 29 of $A L K$. Based on the targeted NGS data, the rearrangement retained the ALK tyrosine kinase region 


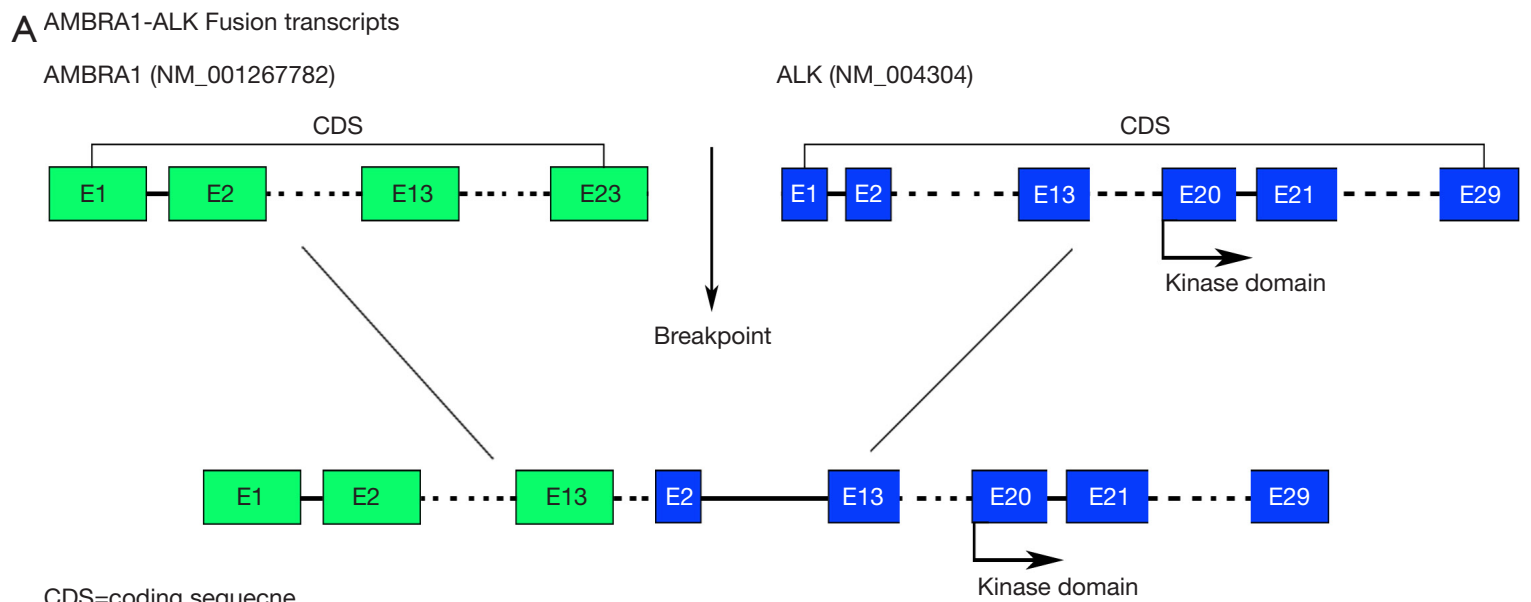

CDS=coding sequecne

B

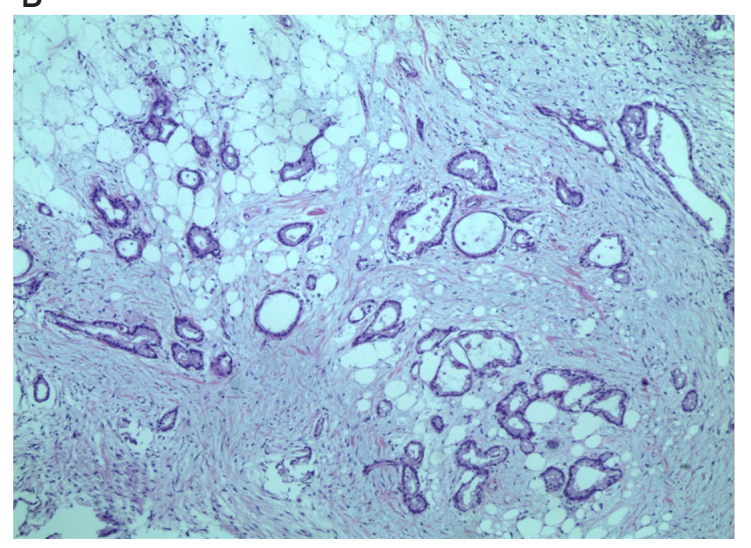

C

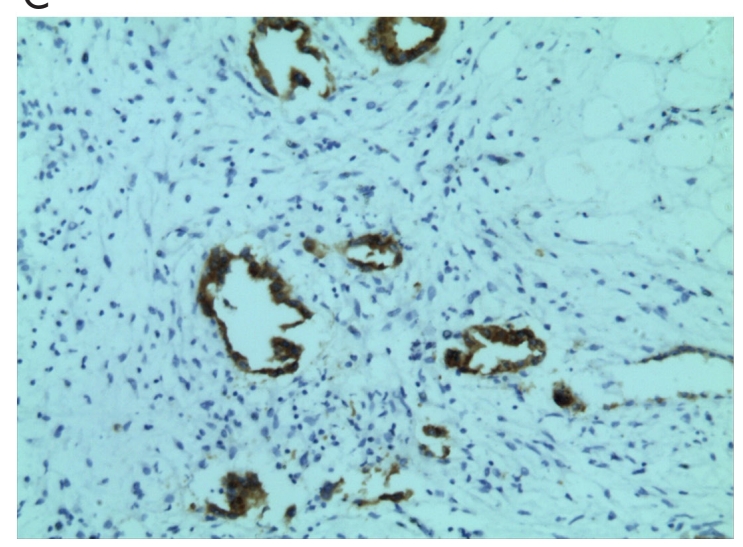

Figure 2 Gene structure and pathological evaluation. (A) Illustration of the gene rearrangement of AMBRA1-ALK; (B) Hematoxylin and eosin staining; (C) immunohistochemical staining (Ventana) of ALK expression. Magnification: 100×. AMBRA1, autophagy/Beclin-1 regulator 1; ALK, anaplastic lymphoma kinase.

that imparts sensitivity to ALK inhibitor crizotinib. IHC confirmed her ALK protein overexpression status. Since the only genetic alteration detected from her tissue sample was $A M B R A 1-A L K$ genomic rearrangement, we speculate that this fusion induced ALK protein overexpression. With the critical roles of both AMBRA1 and ALK in regulating cellular homeostasis, $A M B R A 1-A L K$ fusion may possibly contribute to the deregulation of autophagy due to AMBRA1 haploinsufficiency and induction of aberrant cell proliferation mediated by constitutive activation of ALK. AMBRA1-ALK fusion might potentially be the oncogenic driver for the development of GBC in our patient and also explain her robust clinical response to crizotinib therapy. Further in vitro and in vivo studies are required to confirm the oncogenicity of AMBRA1-ALK in GBC and its sensitivity to ALK inhibitors.

In NSCLC, targeting of non-EML4 ALK fusions that retained the tyrosine kinase domain, including $W D P C P$ $A L K, N C O A 1-A L K$, and CUX1-ALK, with crizotinib has resulted in partial response (27-29). The success of the ALK inhibitor crizotinib in improving clinical outcomes in patients with $A L K$-rearranged advanced NSCLC prompted us to administer crizotinib at the standard dose approved for treatment in advanced NSCLC to our patient with GBC harboring $A M B R A 1-A L K$ rearrangement. Two months of crizotinib therapy resulted in significant shrinkage of the primary lesions, indicating effective targeting of aberrant ALK signaling driven by $A M B R A 1-A L K$ with crizotinib. This finding also suggests that a subset of GBC is driven by ALK and that ALK could serve as a potential biomarker in targeted therapy for GBC.

The use of NGS for exploring potentially actionable mutations in our patient paved the way for administering crizotinib, which improved our patient's quality of life and 
prolonged her survival after traditional chemotherapy had failed. This indicates that NGS could be considered for treatment decisions in patients with GBC.

As this is only a single case report, more research is needed to further understand the role of ALK in driving GBC tumorigenesis and the potential use of ALK inhibitors in the treatment of patients with ALK-positive GBC. Our case report is also limited by the lack of in vitro functional assay for $A M B R A 1-A L K$ and its sensitivity profile to various ALK inhibitors.

In summary, we provide the first clinical evidence of crizotinib efficacy in a patient with GBC harboring a non$E M L 4 A L K$ fusion. Our case report identified a novel $A M B R A 1-A L K$ and its clinical sensitivity to crizotinib therapy, which indicates that a subset of GBC might be driven by aberrant ALK signaling and ALK targeted therapy may represent a new treatment option for patients with advanced GBC. However, more clinical studies are needed to explore the mechanism of ALK in driving GBC and validate the efficacy of ALK inhibitors in patients with ALK-positive GBC. Moreover, our case report contributes an incremental step in understanding the genetic heterogeneity in GBC and provides clinical evidence of the utility of next-generation sequencing in exploring actionable mutations to expand treatment choices in rare solid tumors including GBC.

\section{Acknowledgments}

Funding: None.

\section{Footnote}

Reporting Checklist: The authors have completed the CARE reporting checklist. Available at http://dx.doi.org/10.21037/ atm-20-1007

Peer Review File: Available at http://dx.doi.org/10.21037/ atm-20-1007

Conflicts of Interest: All authors have completed the ICMJE uniform disclosure form (available at http://dx.doi. org/10.21037/atm-20-1007). AL and XM report that they are employees of Burning Rock Biotech. The other authors have no conflicts of interest to declare.

Ethical Statement: The authors are accountable for all aspects of the work in ensuring that questions related to the accuracy or integrity of any part of the work are appropriately investigated and resolved. All procedures performed in studies involving human participant were in accordance with the ethical standards of the institutional and national research committees, and with the Helsinki Declaration (as revised in 2013). The patient provided written informed consent for the publication of this report.

Open Access Statement: This is an Open Access article distributed in accordance with the Creative Commons Attribution-NonCommercial-NoDerivs 4.0 International License (CC BY-NC-ND 4.0), which permits the noncommercial replication and distribution of the article with the strict proviso that no changes or edits are made and the original work is properly cited (including links to both the formal publication through the relevant DOI and the license). See: https://creativecommons.org/licenses/by-nc-nd/4.0/.

\section{References}

1. Maplanka C. Gallbladder cancer, treatment failure and relapses: the peritoneum in gallbladder cancer. J Gastrointest Cancer 2014;45:245-55.

2. Misra S, Chaturvedi A, Misra NC, et al. Carcinoma of the gallbladder. Lancet Oncol 2003;4:167-76.

3. Zhao DY, Lim KH. Current biologics for treatment of biliary tract cancers. J Gastrointest Oncol 2017;8:430-40.

4. Andren-Sandberg A. Molecular biology of gallbladder cancer: potential clinical implications. N Am J Med Sci 2012;4:435-41.

5. Javle M, Rashid A, Churi C, et al. Molecular characterization of gallbladder cancer using somatic mutation profiling. Hum Pathol 2014;45:701-8.

6. Sicklick JK, Fanta PT, Shimabukuro K, et al. Genomics of gallbladder cancer: the case for biomarker-driven clinical trial design. Cancer Metastasis Rev 2016;35:263-75.

7. Chong DQ, Zhu AX. The landscape of targeted therapies for cholangiocarcinoma: current status and emerging targets. Oncotarget 2016;7:46750-67.

8. Weinberg BA, Xiu J, Lindberg MR, et al. Molecular profiling of biliary cancers reveals distinct molecular alterations and potential therapeutic targets. J Gastrointest Oncol 2019;10:652-62.

9. Wang S, Chen R, Tang Y, et al. Comprehensive Genomic Profiling of Rare Tumors: Routes to Targeted Therapies. Front Oncol 2020;10:536.

10. Lee J, Park SH, Chang HM, et al. Gemcitabine and oxaliplatin with or without erlotinib in advanced biliary- 
tract cancer: a multicentre, open-label, randomised, phase 3 study. Lancet Oncol 2012;13:181-8.

11. Peck J, Wei L, Zalupski M, et al. HER2/neu may not be an interesting target in biliary cancers: results of an early phase II study with lapatinib. Oncology 2012;82:175-9.

12. Malka D, Cervera P, Foulon S, et al. Gemcitabine and oxaliplatin with or without cetuximab in advanced biliarytract cancer (BINGO): a randomised, open-label, noncomparative phase 2 trial. Lancet Oncol 2014;15:819-28.

13. Iyer RV, Pokuri VK, Groman A, et al. A Multicenter Phase II Study of Gemcitabine, Capecitabine, and Bevacizumab for Locally Advanced or Metastatic Biliary Tract Cancer. Am J Clin Oncol 2018;41:649-55.

14. Pellino A, Loupakis F, Cadamuro M, et al. Precision medicine in cholangiocarcinoma. Transl Gastroenterol Hepatol 2018;3:40.

15. Nitta T, Sato Y, Ren XS, et al. Autophagy may promote carcinoma cell invasion and correlate with poor prognosis in cholangiocarcinoma. Int J Clin Exp Pathol 2014;7:4913-21.

16. Shaw AT, Kim DW, Nakagawa K, et al. Crizotinib versus chemotherapy in advanced ALK-positive lung cancer. N Engl J Med 2013;368:2385-94.

17. Carpenter EL, Mosse YP. Targeting ALK in neuroblastoma--preclinical and clinical advancements. Nat Rev Clin Oncol 2012;9:391-9.

18. Gambacorti Passerini C, Farina F, Stasia A, et al. Crizotinib in advanced, chemoresistant anaplastic lymphoma kinase-positive lymphoma patients. J Natl Cancer Inst 2014;106:djt378.

19. Roskoski R, Jr. Anaplastic lymphoma kinase (ALK): structure, oncogenic activation, and pharmacological inhibition. Pharmacol Res 2013;68:68-94.

20. Cui JJ, Tran-Dube M, Shen H, et al. Structure based drug design of crizotinib (PF-02341066), a potent and selective dual inhibitor of mesenchymal-epithelial transition factor
(c-MET) kinase and anaplastic lymphoma kinase (ALK). J Med Chem 2011;54:6342-63.

21. You MS, Ryu JK, Choi YH, et al. Therapeutic outcomes and prognostic factors in unresectable gallbladder cancer treated with gemcitabine plus cisplatin. BMC Cancer 2019;19:10.

22. Shu X, Dong Z, Shu S. AMBRA1-mediated autophagy and apoptosis associated with an epithelial-mesenchymal transition in the development of cleft palate induced by all-trans retinoic acid. Ann Transl Med 2019;7:128.

23. Cianfanelli V, De Zio D, Di Bartolomeo S, et al. Ambra 1 at a glance. J Cell Sci 2015;128:2003-8.

24. Cianfanelli V, Fuoco C, Lorente M, et al. AMBRA1 links autophagy to cell proliferation and tumorigenesis by promoting c-Myc dephosphorylation and degradation. Nat Cell Biol 2015;17:20-30.

25. Becher J, Simula L, Volpe E, et al. AMBRA1 Controls Regulatory T-Cell Differentiation and Homeostasis Upstream of the FOXO3-FOXP3 Axis. Dev Cell 2018;47:592-607.e6.

26. Chiang NJ, Hsu C, Chen JS, et al. Expression levels of ROS1/ALK/c-MET and therapeutic efficacy of cetuximab plus chemotherapy in advanced biliary tract cancer. Sci Rep 2016;6:25369.

27. He Z, Wu X, Ma S, et al. Next-generation Sequencing Identified a Novel WDPCP-ALK Fusion Sensitive to Crizotinib in Lung Adenocarcinoma. Clin Lung Cancer 2019;20:e548-51.

28. Cao Q, Liu Z, Huang Y, et al. NCOA1-ALK: a novel ALK rearrangement in one lung adenocarcinoma patient responding to crizotinib treatment. Onco Targets Ther 2019;12:1071-4.

29. Zhang M, Wang Q, Ding Y, et al. CUX1-ALK, a Novel ALK Rearrangement That Responds to Crizotinib in NonSmall Cell Lung Cancer. J Thorac Oncol 2018;13:1792-7.
Cite this article as: Zhou Y, Lizaso A, Mao X, Yang N, Zhang Y. Novel AMBRA1-ALK fusion identified by nextgeneration sequencing in advanced gallbladder cancer responds to crizotinib: a case report. Ann Transl Med 2020;8(17):1099. doi: 10.21037/atm-20-1007 